\title{
Originales
}

\section{Variaciones en la calidad de vida relacionada con la salud en los pacientes críticos}

\author{
S. IRIBARREN-DIARASARRI ${ }^{a}$, F. AIZPURU-BARANDIARAN ${ }^{b}$, T. MUÑOZ-MARTÍNEZa ${ }^{a}$ J.L. DUDAGOITIA- \\ OTAOLEA a Á. CASTAÑEDA-SÁEZa , M. HERNÁNDEZ-LÓPEZa, S. MARTÍNEZ-ALUTIZa , C. VINUESA-LOZANOa \\ Y N. ARETXABALA-KORTAJARENA ${ }^{a}$
}

aUnidad de Medicina Intensiva. Hospital Txagorritxu. Vitoria. Álava. España.

bUnidad de Investigación. Hospital Txagorritxu. Vitoria. Álava. España.

Objetivo. Analizar las variaciones de la calidad de vida relacionada con la salud (CVRS) a los 6 meses del alta de la unidad de medicina intensiva (UMI)y los factores que se asocian a estas variaciones.

Diseño. Estudio prospectivo de cohortes.

Ámbito. UMI médico-quirúrgica de 14 camas.

Pacientes. Fueron 247 pacientes ingresados en UMI más de $24 \mathrm{~h}$, con un seguimiento de 6 meses. Se excluyó a los pacientes con síndrome coronario agudo y los que ingresaban solamente para seguimiento.

Intervención. Se realizó una encuesta de CVRS desarrollada por el grupo Proyecto para el Análisis Epidemiológico del Enfermo Crítico sobre su estado previo y a los 6 meses del alta de la UMI.

Resultados. La CVRS se deteriora pasando de una mediana de 3 puntos a $6(p<0,001)$. El análisis multivariable muestra menor deterioro en la CVRS en pacientes con antecedentes registrados por el APACHE II (coeficiente de regresión [CR] $=-1,4$; intervalo de confianza [IC] del $95 \%,-2,5$ a $-0,2 ; p=0,02)$ o una CVRS $\geq 10$ puntos (CR $=-4,4$; IC del 95\%, -5,9 a -2,8; $p<0,001$ ) y mayor deterioro en pacientes politraumatizados (CR = 1,9; IC del $95 \%, 0,6-3,3 ; p<0,01)$, con insuficiencia renal (CR = 3,9; IC del 95\%, 1,9-5,9; p < 0,001) o estancia en $\mathrm{UMI}$ > 10 días (CR = 1,9; IC del 95\%, 0,6-3,2; $p$ $=0,004)$.
Correspondencia: Dr. S. Iribarren Diarasarri.

Hospital Txagorritxu.

Jose Atxotegi, s/n. 01009 Vitoria. Álava. España.

Correo electrónico: SEBASTIAN.IRIBARRENDIARASARRI@

osakidetza.net

Manuscrito aceptado el 27-10-2008.
Conclusiones. La mayoría de los pacientes muestran un deterioro de la CVRS. Los pacientes con enfermedades crónicas o con peor CVRS previa que han sobrevivido a los 6 meses muestran menor deterioro que los politraumatizados 0 con insuficiencia renal o estancias prolongadas en UMI.

PALABRAS ClAVE: Calidad de vida. Medicina intensiva. Factores pronósticos.

\section{VARIATIONS IN HEALTH-RELATED QUALITY OF LIFE IN CRITICAL PATIENTS}

Objective. To determine the changes in the health-related quality of life (HRQOL) six months after discharge from the ICU and the conditions associated to them.

Design. A prospective cohort study.

Setting. 14 beds medical-surgical intensive care unit (ICU).

Patients. A total of 247 patients admitted to our ICU for more than 24 hours with a follow-up of 6 months were study. Those admitted with acute coronary syndrome or for monitoring purposes were excluded.

Intervention. A quality of life survey was conducted using the score developed by the PAEEC group (project of the epidemiological analysis of critical illness) to assess HRQOL before ICU admission and 6 months after discharge.

Results. The HRQOL deteriorated, going from a median value of 3 to 6 ( $p<0.001)$. The multivariate analysis showed less deterioration of HRQOL in patients with chronic health conditions registered on the APACHE-II score (regression coefficient [RC] = -1.4; 95\% Cl, -2.5 to $-0.2 ; p<0.02)$ and in those with a HRQOL $\geq 10$ points $(R C=-4,4 ; 95 \%$ 
$\mathrm{Cl},-5.9$ to $-2.8 ; \mathrm{p}<0.001)$. There was more deterioration in polytraumatized patients $(R C=1.9$; 95\% Cl, 0.6-3.3; $\mathrm{p}=0.01$ ) or with renal failure (RC $=3.9 ; 95 \% \mathrm{CI}, 1.9-5.9 ; \mathrm{p}<0.001$ ) or in those with a stay duration longer than 10 days $(R C=1.9 ; 95 \%$ $\mathrm{Cl}, 0.6-3.2 ; \mathrm{p}<0.001$ ).

Conclusions. Most patients experience deterioration of HRQOL. Patients with chronic diseases or with worst previous HRQOL who survive 6 months experience less deterioration of HRQOL than those who are polytraumatized or have renal failure or a longer ICU stay.

KEY WORDS: Quality of life. Intensive care. Prognostic factors.

\section{INTRODUCCIÓN}

Un porcentaje muy elevado de los pacientes que ingresan en las unidades de medicina intensiva (UMI) son mayores de 70 años ${ }^{1}$, y no lo hacen tanto por procesos agudos como por descompensaciones de procesos crónicos, como la cardiopatía isquémica, la enfermedad pulmonar obstructiva crónica (EPOC), la hepatopatía crónica, etc ${ }^{2}$. Esto hace que no sea suficiente hablar de mortalidad para describir los resultados de nuestra actividad, es necesario también conocer el pronóstico funcional de estos pacientes ${ }^{3}$. En definitiva, se ha hecho necesario investigar sobre la calidad de vida relacionada con la salud (CVRS) de nuestros pacientes. La mayoría de los autores entienden que ésta es la suma de las funciones físicas y psicológicas, la capacidad para satisfacer sus necesidades sociales junto con la propia percepción de su situación ${ }^{4}$.

Los estudios relacionados con la CVRS de pacientes ingresados en UMI son cada vez más frecuentes en la literatura médica; sin embargo, es todavía un aspecto poco estudiado si se lo compara con otros ${ }^{5}$. No obstante, la CVRS previa y la esperada tras el alta son uno de los factores que más tienen en cuenta los profesionales a la hora de aceptar un ingreso en una UMI y aplicar un tratamiento de soporte vital completo ${ }^{6}$.

El objetivo del presente trabajo es conocer las variaciones que se producen en la CVRS 6 meses después del paso por una UMI, así como la asociación de las variaciones con la situación previa del paciente (CVRS previa y enfermedades crónicas), los grupos diagnósticos, la gravedad del proceso (APACHE II, sepsis y disfunciones orgánicas) que causa su ingreso en la UMI, y la duración de la estancia en la UMI.

\section{PACIENTES Y MÉTODO}

Se trata de un estudio prospectivo de cohortes. El ámbito del estudio es la población atendida por la UMI del Hospital Txagorritxu, un hospital de segundo nivel, durante los años 1999 y 2000. Esta unidad consta de 14 camas. Se incluyó en la cohorte a los pacientes mayores de 18 años que ingresaron en la UMI y consintieron participar en el estudio. Se excluyó a los pacientes ingresados por síndrome coronario agudo o con estancias $<24 \mathrm{~h}$, ingresados exclusivamente para la supervisión de un tratamiento y los reingresos en la unidad. Se analizaron las siguientes variables con el fin de estudiar su asociación a las variaciones en la CVRS: variables demográficas como edad y sexo; enfermedades crónicas previas como diabetes mellitus, neumopatía crónica obstructiva o restrictiva ${ }^{7}$, insuficiencia renal crónica (creatinina $>1,4 \mathrm{mg} / \mathrm{dl}$ ), depresión, cirrosis hepática y antecedentes considerados en el APACHE $\mathrm{II}^{8}$ (cirrosis, clase funcional IV de la New York Heart Association, EPOC grave, hipertensión pulmonar, diálisis crónica, inmumosupresión), grupos diagnósticos, indicadores de gravedad en las primeras $24 \mathrm{~h}$ (APACHE II), incidencia de sepsis ${ }^{9}$ al ingreso o durante su estancia en la UMI, indicadores de gravedad del síndrome de disfunción multiorgánica utilizando el SOFA (Sequential Organ Failure Assessment ${ }^{10}$ que contempla 6 sistemas: hemodinámico, respiratorio, renal, hepático, hematológico y neurológico. Se registró el SOFA de forma diaria utilizándose para el análisis la máxima puntuación alcanzada durante un día (SOFA máximo) a lo largo de la estancia del paciente en la UMI. Consideramos disfunción grave o fracaso de un sistema una puntuación de 3-4 puntos en el sistema correspondiente en la escala SOFA. Consideramos fracaso multiorgánico el fracaso de dos o más sistemas ${ }^{11}$. Se consideró también la realización de traqueotomía y la duración de la estancia en la UMI.

Para evaluar la CVRS se empleó el Cuestionario de Calidad de Vida para pacientes Críticos diseñado y validado en España por el Grupo PAEEC (Proyecto para el Análisis Epidemiológico del Enfermo Crítico $)^{12}$. Este cuestionario analiza tres dimensiones: actividades fisiológicas básicas ( 0 a 9 puntos), actividades de la vida diaria que abarca actividad física, laboral y social ( 0 a 15 puntos) y el estado emocional (0 a 5 puntos). Consta de 15 cuestiones con su puntuación correspondiente, de tal forma que la puntuación total puede ir desde 0 , que traduce una calidad de vida normal o sin limitaciones, hasta 29 puntos, máximo deterioro de la calidad de vida.

La encuesta fue realizada por tres médicos, que supervisaban las respuestas y aclaraban las dudas del paciente o representante, y se realizó al ingreso en la UMI tomando como referencia la situación existente en el mes previo. Si el paciente no era capaz de contestar al cuestionario se realizaba a un familiar que conviviese con el paciente, dado de que cuestionario cumple el criterio de reproducibilidad (coeficiente de correlación $>0,90)^{12}$.

Se repitió el cuestionario a los 6 meses del alta de la UMI a todos los pacientes supervivientes o a sus familiares en caso de incapacidad de los primeros. El cuestionario se completaba mediante entrevista personal o telefónica. Dentro de las limitaciones propias del horario laboral, el paciente establecía la cita de la entrevista, con el fin de disminuir las pérdidas en el seguimiento.

El cambio en calidad de vida desde la situación previa al ingreso hasta el final del seguimiento (6 meses) se evaluó mediante el test de Wilcoxon para datos apareados. La asociación entre el cambio observado en la CVRS (CVRS a los 6 meses-CVRS al ingreso) con las distintas variables demográficas o clínicas estudiadas se examinó mediante la prueba de 
la t de Student (datos independientes) o la prueba no paramétrica de Mann-Whitney cuando no se pudo asumir la distribución normal o para analizar subgrupos de pequeño tamaño. La capacidad predictiva de cada variable estudiada sobre la variación en la CVRS se determinó mediante un modelo de regresión lineal. Las variables estancia en UMI y CVRS previa, de distribución marcadamente asimétrica, se categorizaron según los cuartiles de la distribución para facilitar su inclusión en este análisis. Se ajustó el modelo en función de quién había contestado a la encuesta inicial, el propio paciente o un allegado. Se consideró un nivel de significación en $\mathrm{p}<0,05$. El análisis se realizó con el paquete estadístico SPSS versión 14.0.

\section{RESULTADOS}

El flujo de pacientes reclutados se muestra en la figura $1 ; 19$ pacientes que cumplían criterios de inclusión se perdieron por haber sido dados de alta o fallecer antes de poder realizar la encuesta de calidad de vida. Los grupos diagnósticos de la cohorte y las características que definen a los pacientes se muestran en las tablas 1 y 2 respectivamente.

La mortalidad en la UMI fue del 14,6\% (55 pacientes) y la hospitalaria, del 24,9\% (94 pacientes). A los 6 meses del alta de la UMI sobrevivían 252 $(66,8 \%)$. No se pudo localizar a 5 pacientes, por lo que se realizó la segunda entrevista a 247 (98,8\%).

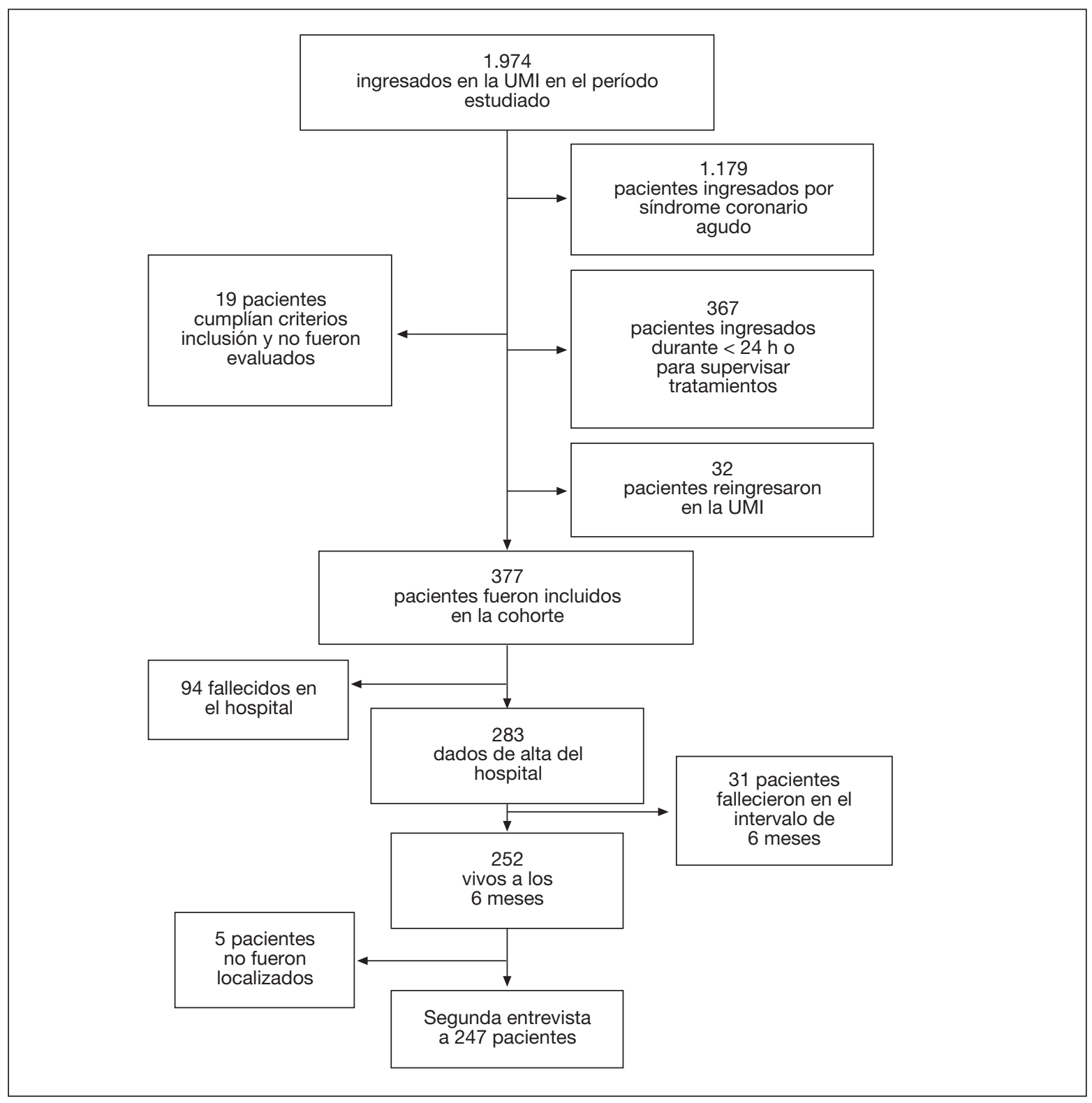

Figura 1. Flujo de la cohorte. 
TABLA 1. Grupos diagnósticos de la cohorte de pacientes incluidos en el estudio

\begin{tabular}{|l|c|c|}
\hline & $\begin{array}{c}\text { Total de pacientes } \\
(\mathrm{n}=377), \mathrm{n}(\%)\end{array}$ & $\begin{array}{c}\text { Pacientes con } \\
\text { seguimiento a los } \\
6 \text { meses }(\mathrm{n}=247), \\
\mathrm{n}(\%)\end{array}$ \\
\hline Médicos & $202(54)$ & $121(49)$ \\
Neumología & $104(27,6)$ & $54(21,9)$ \\
Neurología & $21(5,6)$ & $15(6,1)$ \\
Sepsis & $20(5,3)$ & $12(4,9)$ \\
Cardiovascular & $20(5,3)$ & $15(6,1)$ \\
Traumatología & $14(3,7)$ & $10(4)$ \\
Digestivo & $12(3,2)$ & $9(3,6)$ \\
Renal & $5(1,3)$ & $2(0,8)$ \\
Otros & $6(2,1)$ & $4(1,6)$ \\
Quirúrgicos & $175(46)$ & $126(51)$ \\
Cirugía digestiva & $96(25,5)$ & $66(26,7)$ \\
Traumatología & $28(7,4)$ & $23(9,3)$ \\
Cirugía vascular & $26(6,9)$ & $19(7,7)$ \\
Urología & $11(2,9)$ & $6(2,4)$ \\
Otros & $14(3,7)$ & $12(4,8)$ \\
\hline
\end{tabular}

TABLA 2. Características de los pacientes incluidos en el estudio

\begin{tabular}{|l|c|c|}
\hline & $\begin{array}{c}\text { Total de pacientes } \\
(\mathrm{n}=377)\end{array}$ & $\begin{array}{c}\text { Seguimiento a 6 } \\
\text { meses }(\mathrm{n}=247)\end{array}$ \\
\hline Varones & $249(66)$ & $158(64)$ \\
Edad (años) & $62 \pm 16$ & $59 \pm 17$ \\
Neumopatía crónica & $107(28)$ & $64(26)$ \\
Diabetes mellitus & $45(12)$ & $30(12)$ \\
Depresión & $28(7)$ & $16(6)$ \\
Insuficiencia renal & $34(9)$ & $17(7)$ \\
$\quad$ crónica & $21(6)$ & $12(5)$ \\
Cirrosis & $124(33)$ & $65(26)$ \\
APACHE II & & \\
$\quad$ antecedentes) & $5,3 \pm 4,9$ & $4,1 \pm 4,4$ \\
CVRS previa & $16,9 \pm 7,5$ & $14,7 \pm 6,7$ \\
APACHE II & $32(8)$ & $15(6)$ \\
Traqueotomizados & $211(56)$ & $121(49)$ \\
Sepsis al ingreso & & \\
$\quad$ o durante el ingreso & & \\
en UMI & & \\
\hline
\end{tabular}

Resultados expresados como n (\%) o media \pm desviación estándar.
La encuesta de calidad de vida previa se realizó en 165 (67\%) casos al mismo paciente y en 82 (33\%) a un familiar que convivía con el paciente, dado que éste era incapaz. A los 6 meses, la entrevista se realizó al mismo paciente en el $98 \%$ de los casos y en el resto a un familiar conviviente. El 77,7\% de las entrevistas fueron presenciales y las restantes fueron telefónicas.

En la figura 2 se observan las variaciones en la CVRS general y en sus tres subescalas, todas ellas estadísticamente significativas $(\mathrm{p}<0,001)$. Aunque se aprecia un deterioro de la calidad de vida general y de sus distintas subescalas, desglosando los datos hallamos que la CVRS general empeoró en $148(60 \%)$ pacientes, permaneció igual en $50(20 \%)$ y mejoró en 49 (20\%). La tabla 3 expresa la distribución de las respuestas a cada una de las cuestiones que recoge la encuesta de CVRS en los dos momentos estudiados.

No se observó diferencia en la variación en la CVRS en función del sexo (varones, $2,7 \pm 4,3$; mujeres, 1,8 \pm $5 ; p=0,11)$ o la edad $(r=0,05 ; p=0,3) . \operatorname{Los} 65(26,3 \%)$ pacientes que sufrían enfermedades crónicas consideradas en el APACHE II mostraron un menor deterioro de su CVRS que aquellos que no $(1,2 \pm 4,6$ frente a 2,8 $\pm 4,5 ; p=0,013)$. Sin embargo, no encontramos diferencias en la variación de la CVRS en relación con tener o no alguna de las siguientes enfermedades crónicas: neumopatía crónica (64 pacientes, $1,9 \pm 4,5$ frente a $2,6 \pm 4,5 ; p=0,3$ ), diabetes mellitus (30 pacientes, $1,6 \pm 3,2$ frente a $2,4 \pm 4,7 ; \mathrm{p}=0,5)$, depresión (16 pacientes, $1,2 \pm 5,2$ frente a $2,5 \pm 4,5 ; \mathrm{p}=0,3$ ) o insuficiencia renal crónica (17 pacientes, 4,2 $\pm 4,9$ frente a $2,3 \pm 4,5 ; \mathrm{p}=0,1)$. La puntuación total del índice APACHE II tampoco se mostró asociada al cambio en calidad de vida $(r=0,03 ; p=0,48)$.

La CVRS previa tiene una asociación inversa con la variación en la CVRS. Los pacientes en el primer cuartil de la distribución (CVRS $\leq 1)$ empeoraron de media 3,3 puntos (intervalo de confianza [IC] del $95 \%$, 2,5-4,2) en los primeros 6 meses al alta hospitalaria. El empeoramiento fue menor en los del segundo ( 3,05 ; IC del 95\%, 2,4-4,06) y el tercer cuartil $(2,4$; IC del 95\%, 1,3-3,5). Por último, los pacientes con peor CVRS

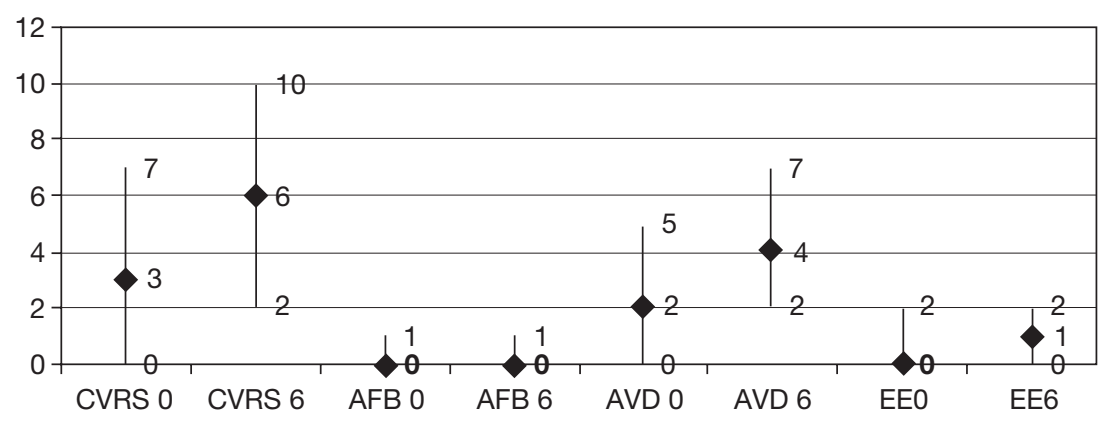

Figura 2. Variaciones en la calidad de vida relacionada con la salud (CVRS) general y en las tres dimensiones o subescalas del cuestionario. AFB 0: actividades fisiológicas básicas previas; AFB 6: actividades fisiológicas básicas a los 6 meses; AVD 0: actividades de la vida diaria previas; AVD 6: actividades de la vida diaria a los 6 meses; CVRS 0: índice general previo; CVRS 6: índice general a los 6 meses; EE 0: estado emocional previo: EE 6: estado emocional a los 6 meses. El gráfico expresa las medianas y los percentiles 25 y 75. Todas las variaciones muestran $p<0,001$. 
TABLA 3. Variaciones en la CVRS en los 247 pacientes a los que se pudo realizar el seguimiento a los 6 meses

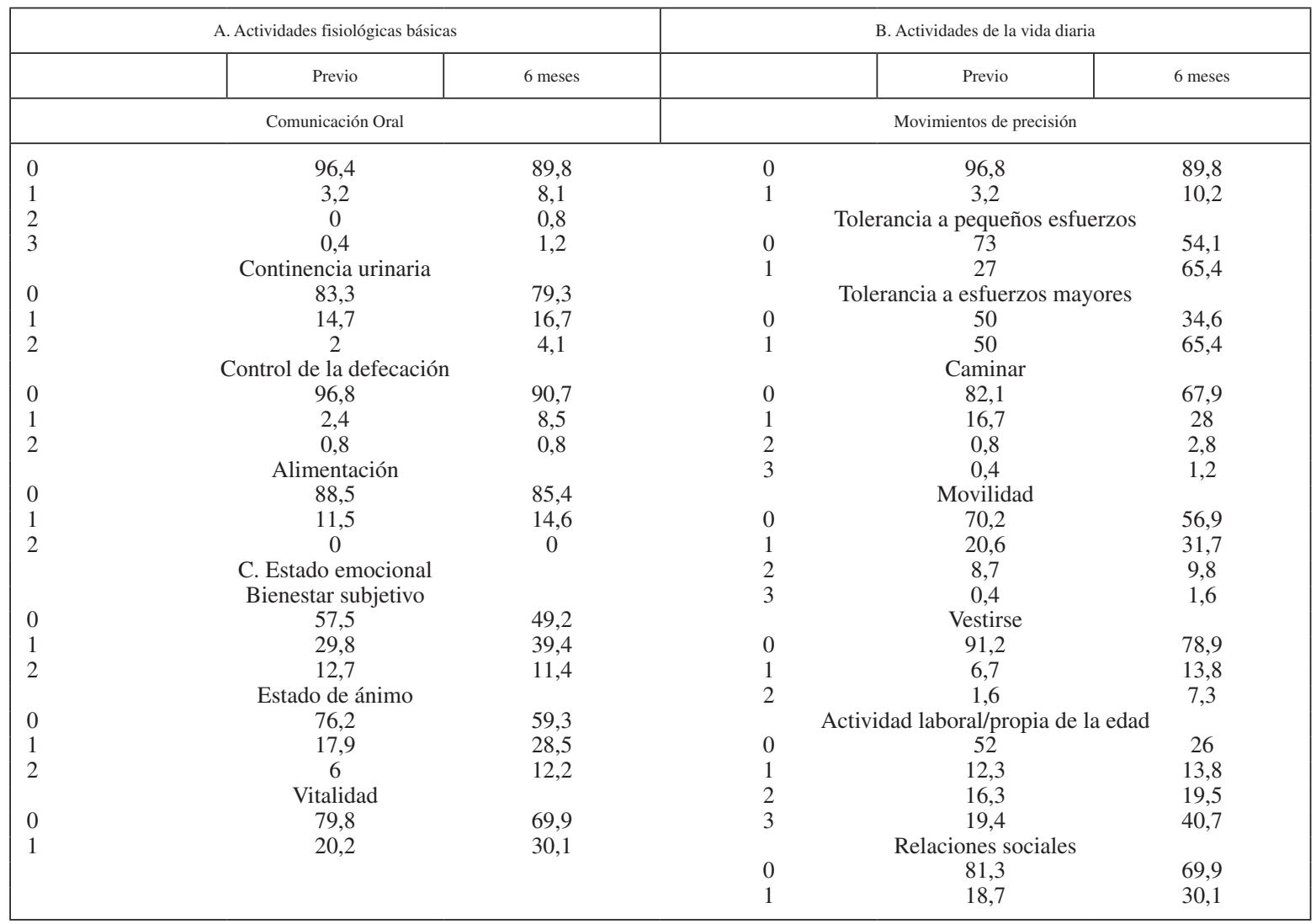

Porcentaje de respuestas obtenidas a cada pregunta del cuestionario de CVRS, al ingreso en UMI y 6 meses tras el alta. El valor 0 equivale a normalidad y puntuaciones mayores indican mayor deterioro.

$(\mathrm{CVRS} \leq 10)$ mejoraron en 1,7 puntos (IC del 95\%, $-3,7$ a 0,35$)$ a los 6 meses $\left(p_{\text {tendencia }}<0,001\right)$.

La CVRS empeoró más en los pacientes sépticos que en los no sépticos (121 pacientes, $3 \pm 4,4$ frente a $1,8 \pm 4,8 ; \mathrm{p}=0,036)$, en los politraumatizados (33 pacientes, 4,9 $\pm 3,6$ frente a 2,02 $\pm 4,6 ; p=0,001) y$, aunque sin significación estadística, entre los traquetomizados frente a los que no (15 pacientes, 4,5 $\pm 5,4$ frente a $2,3 \pm 4,5 ; p=0,07)$. No se observó asociación entre la variación de la CVRS y la causa, médica (121 pacientes) o quirúrgica (126 pacientes) del ingreso $(2,3 \pm 4,7$ frente a $2,5 \pm 4,5 ; \mathrm{p}=0,7)$ ni entre los que sufrieron fracaso multiorgánico frente a los que no (63 pacientes, 2,7 \pm 4,4 frente a 2,3 $\pm 4,7 ; \mathrm{p}=0,57$ ).

En cuanto a los distintos órganos o sistemas analizados de forma independiente, el único asociado a mayor deterioro de la CVRS fue la insuficiencia renal (19 pacientes; $6 \pm 4,7$ frente a $2,1 \pm 4,4 ; p<0,001)$. No se observó asociación en relación con insuficiencia respiratoria (86 pacientes; $2,6 \pm 4,3$ frente a $2,3 \pm 4,7 ; \mathrm{p}=$ $0,6)$, hemodinámico (80 pacientes; $2,3 \pm 4,2$ frente a $2,4 \pm 4,7 ; \mathrm{p}=0,9)$, hematológico (13 pacientes; 4,6 \pm $4,7$ frente a $2,3 \pm 4,6 ; \mathrm{p}=0,10)$, neurológico ( 8 pacientes, $6,7 \pm 7,7$ frente a $2,2 \pm 4,4 ; p=0,14)$ o hepático $(3$ pacientes, $1 \pm 7,2$ frente a $2,4 \pm 4,5 ; \mathrm{p}=0,6)$. De los 19 pacientes con fracaso renal, a los 6 meses 9 no habían normalizado la función renal y 5 estaban en diálisis.

Por último, la duración de estancia en la UMI, categorizada en cuartiles, también muestra asociación lineal directa $\left(\mathrm{p}_{\text {tendencia }}=0,001\right)$ con el empeoramiento de la CVRS, si bien la asociación es significativa sólo para el cuarto cuartil (> 10 días de estancia) si tomamos como referencia el primero ( $\leq 2$ días de estancia) (coeficiente de regresión $=2,9 ; \mathrm{p}<0,001)$. El empeoramiento neto fue de 1,7 puntos en el primero y el segundo cuartiles (3-4 días), 2,1 puntos en el tercer cuartil (5-10 días) y 4,5 puntos en el cuartil superior. Los pacientes con estancias en UMI superiores a 10 días eran predominantemente pacientes quirúrgi$\cos (37 \%)$ y respiratorios $(30 \%)$.

El análisis multivariable (tabla 4) muestra que sufrir un politraumatismo o tener insuficiencia renal o una estancia en la UMI > 10 días se asocian de manera independiente con un mayor empeoramiento de la CVRS a los 6 meses.

Por el contrario, los pacientes que presentan enfermedades crónicas incluidas en el APACHE II o una CVRS previa $\geq 10$ puntos muestran un menor deterioro de la CVRS a los 6 meses del alta de la UMI que aquellos sin antecedentes incluidos en el APACHE II 
TABLA 4. Análisis multivariable mediante modelo de regresión lineal múltiple

\begin{tabular}{|c|c|c|}
\hline Variable & $\begin{array}{l}\text { Coeficiente } \\
\text { de regresión } \\
\text { (IC del 95\%) }\end{array}$ & $p$ \\
\hline Constante & $2,6(1,3-3)$ & \\
\hline $\begin{array}{l}\text { APACHE II- } \\
\text { antecedentes }\end{array}$ & $-1,4(-2,5 \mathrm{a}-0,2)$ & 0,023 \\
\hline SOFA & & $\mathrm{p}_{\text {tendencia }}$ \\
\hline$\leq 4$ & 1 & \\
\hline $5-8$ & $0,05(-1,4$ a 1,5$)$ & 0,9 \\
\hline$\geq 9$ & $-0,67(-2,7$ a 1,3$)$ & 0,5 \\
\hline Sepsis & $0,2(-0,96$ a 1,47$)$ & 0,67 \\
\hline Insuficiencia renal & $3,9(1,9-5,9)$ & $<0,001$ \\
\hline CVRS previa & & $\mathrm{p}_{\text {tendencia }}$ \\
\hline$\leq 1$ & 1 & \\
\hline $2-4$ & $-0,3(-1,7$ a 1,1$)$ & \\
\hline $5-9$ & $-0,28(-1,7$ a 1,1$)$ & \\
\hline$\geq 10$ & $-4,4(-5,9 a-2,8)$ & $<0,001$ \\
\hline Politraumatizado & $1,9(0,6-3,2)$ & 0,014 \\
\hline $\begin{array}{l}\text { Respuesta a primera } \\
\text { entrevista }\end{array}$ & $-0,33(-1,6$ a 0,9$)$ & 0,6 \\
\hline $\begin{array}{l}\text { Estancia en UMI } \\
\leq 2\end{array}$ & 1 & $\mathrm{p}_{\text {tendencia }}$ \\
\hline $3-4$ & $0,05(-1,4$ a 1,5$)$ & 0,9 \\
\hline $5-10$ & $0,5(-1,3$ a 2,3$)$ & 0,6 \\
\hline$>10$ días & $1,9(0,6-3,2)$ & 0,004 \\
\hline
\end{tabular}

o con una $C V R S<10$. El cambio observado era independiente de quién (el propio paciente o un allegado) había contestado a la encuesta inicial.

\section{DISCUSIÓN}

Basándonos en la misma cohorte de pacientes hemos publicado ya resultados sobre mortalidad a 1 año y calidad de vida en pacientes con EPOC, así como sobre limitación del esfuerzo terapéutico ${ }^{13,14}$. Sin embargo, consideramos fundamental conocer el pronóstico de determinado proceso o entidad patológica que no puede quedar limitado a conocer su mortalidad a corto, ni siquiera a largo plazo. El pronóstico comprende también la calidad de vida o estado funcional del paciente. Con el fin de valorar la CVRS se han diseñado múltiples cuestionarios. Nosotros hemos utilizado un cuestionario para pacientes críticos validado en nuestro país, y más recientemente en Italia. Los estudios sobre variaciones en la calidad de vida suelen mostrar frecuentemente dos debilidades: una es la elevada tasa de pérdidas en el seguimiento ${ }^{16-19}, \mathrm{y}$ otra es que en muchos casos no ha habido una valoración previa o un grupo control para poder realizar una comparación ${ }^{16,17}$. Nosotros conseguimos minimizar las perdidas de tal manera que solamente se perdieron $5(2 \%)$ pacientes, y además en la gran mayoría, cerca del $80 \%$, la encuesta se realizó de manera presencial, lo que refuerza la validez interna del estudio.

También está en discusión cuál es el momento de valorar la CVRS tras un proceso grave. De acuerdo con otros autores ${ }^{20,21}$, nosotros optamos por los 6 meses dado que a mayor precocidad restamos posibilidades de recuperación, sobre todo en pacientes politraumatizados, y si la distanciamos demasiado, aumenta el riesgo de que procesos intercurrentes tengan influencia negativa en la CVRS.

Habitualmente las valoraciones de la CVRS se relacionan con distintos grupos diagnósticos, nosotros decidimos que además de estudiar la evolución en determinados grupos era necesario tener en cuenta los antecedentes y la gravedad del proceso que obliga a los pacientes a ingresar en una UMI, porque pensamos que estas variables pueden tener una mayor influencia que la clasificación en un grupo diagnóstico.

Los pacientes que han sobrevivido 6 meses tras el alta hospitalaria muestran mayoritariamente un deterioro de la CVRS. La mediana se duplica (empeora) y pasa de 3 a 6 puntos. Estos resultados concuerdan con los de otros estudios publicados hasta ahora ${ }^{21-26}$, que muestran un grado variable de deterioro de la CVRS previa. Aunque este deterioro se da en los tres aspectos que analiza la encuesta -actividades fisiológicas básicas, actividades de la vida diaria y estado emocional-, es mucho más llamativo en las actividades de la vida diaria, donde la mediana aumenta de 2 a 4 puntos. Analizando cada una de las preguntas de la encuesta, podemos destacar los siguientes hechos: el desarrollo normal de una actividad laboral o propia de la edad pasa del $52 \%$ de los pacientes al $26 \%$, lo que supone una pérdida en valores absolutos del $26 \%$. La tolerancia a pequeños esfuerzos se pierde en un $19 \%$ y en esfuerzos mayores, un $15,4 \%$. La ausencia de limitaciones para caminar, moverse o vestirse sin dificultad cae el 14, el 13 y el $12 \%$ respectivamente y un $11 \%$ de los pacientes revelan un deterioro en sus relaciones sociales. Concuerda con otros estudios el menor deterioro de la sensación subjetiva de bienestar en relación con otros aspectos, solamente decae un $8 \%$; sin embargo, el estado de ánimo empeora en un $16 \%$ de los pacientes. Las actividades fisiológicas básicas (comer, control de esfínteres y alimentación) empeoran sólo ligeramente y están mayoritariamente preservadas en nuestros pacientes. Podemos afirmar, por lo tanto, que aunque hay un deterioro de la CVRS tanto clínica como estadísticamente significativo, la mayoría de los pacientes son autosuficientes para desempeñar sus actividades básicas.

Al analizar los posibles factores asociados a las variaciones en la CVRS, hemos encontrado que la existencia de antecedentes incluidos en el APACHE II se asocia con un menor deterioro de la CVRS previa. En la misma línea, los pacientes que tenían una mala CVRS previa ( $\leq 10$ puntos) muestran también un menor deterioro de su CVRS. En definitiva y de acuerdo con resultados ya publicados por Vázquez-Mata et $\mathrm{al}^{27}$, Wehler et $\mathrm{al}^{28}$ y Cuthberson et $\mathrm{al}^{29}$, entre otros, parece que en los pacientes con un severo deterioro de su CVRS previa o con enfermedades crónicas en estadios avanzados, como las incluidas en el APACHE II, que sobreviven durante 5-12 meses a su ingreso en la UMI, cabe esperar un menor deterioro o incluso una mejoría que en aquellos que partían de una buena CVRS o carecían de enfermedades crónicas avanzadas. Este hecho parece razonable si, junto con que partir de una peor CVRS previa da menor margen de empeorar, lo unimos a que cada vez hay más datos de que el deterio- 
ro severo de la CVRS previa al ingreso en una UMI se asocia con mayor mortalidad a corto-medio plazo ${ }^{13,25,30}$. El hecho de que no encontremos asociación con las distintas enfermedades crónicas evaluadas lo atribuimos a la baja prevalencia de cada una de ellas, en nuestra cohorte, lo que puede dar lugar a un error de tipo II, ya que la muestra no está diseñada para incluir este aspecto. En contra de lo que esperábamos, pero al igual que Clermont et $\mathrm{al}^{31}$, Graf et $\mathrm{al}^{32}$ o Pettilä et $\mathrm{al}^{23}$, no hemos encontrado asociación entre la disfunción multiorgánica, cuantificada mediante el SOFA máximo y las variaciones en la CVRS. Por lo tanto, deberíamos concluir que los pacientes que sobreviven al fracaso multiorgánico no parecen sufrir secuelas que deterioren de forma significativa su CVRS. Sin embargo, la insuficiencia renal, definida como 3-4 puntos en el índice SOFA de este órgano, sí muestra asociación con un deterioro de la CVRS a 6 meses, resultados en la misma línea pueden deducirse de lo publicado por Korkeila et $\mathrm{al}^{33}$ y Capuzzo et al ${ }^{34}$. Esto podría explicarse por la persistencia de insuficiencia renal en la mayoría de los pacientes.

Al igual que Badía et $\mathrm{al}^{26}$, Konopad et $\mathrm{al}^{21}$ y Niskanen et $a^{22}$, también en nuestros pacientes hemos encontrado una asociación entre estancias prolongadas en la UMI > 10 días con un mayor riesgo de deterioro en la CVRS. La polineuropatía del paciente crítico, que no se ha estudiado en el presente trabajo, bien pudiera ser el eslabón que uniera la estancia prolongada con un deterioro de la CVRS, sobre todo teniendo en cuenta que el aspecto que sufre un mayor deterioro es la actividad física ${ }^{35,36}$.

Los pacientes politraumatizados muestran también un deterioro significativo de su CVRS a 6 meses, independiente a las demás variables analizadas, este dato es acorde con lo descrito por otros autores ${ }^{25,26}$.

A la hora de analizar las limitaciones del presente trabajo, cabe destacar que se trata de un trabajo unicéntrico con la limitación que ello supone a la hora de generalizar las conclusiones. La escasa prevalencia de determinadas enfermedades crónicas puede dar lugar a errores de tipo II en el análisis al igual que la baja incidencia de algunas disfunciones orgánicas, como la hematológica, neurológica o hepática. Por otro lado, la imposibilidad de realizar la encuesta al paciente en todos los casos y tener que recurrir a familiares del paciente supone una limitación en muchos casos ineludible en nuestros pacientes s $^{13,25,30,36}$. Por ello elegimos una encuesta validada para poder realizarla a representantes, aunque pudiera considerarse una limitación del estudio el hecho de utilizar solamente una. Además es conocido que la mayor discordancia entre el paciente y su representante se da en los aspectos emocionales de la CVRS, que en el cuestionario utilizado tienen un peso limitado ( 5 puntos sobre 29 posibles) ${ }^{18,36-38}$. Realizar la valoración de la CVRS previa simultáneamente a la de los 6 meses tras el alta habría eliminado este posible sesgo, pero a cambio de aumentar el de recuerdo. Tuvimos en cuenta este problema en el análisis y no encontramos diferencias entre las encuestas contestadas por el paciente y las contestadas por la familia.
Para finalizar, podemos concluir afirmando que los pacientes de la cohorte muestran mayoritariamente un deterioro de su CVRS a los 6 meses del alta de la UMI que se asocia de manera independiente con ser pacientes politraumatizados, la estancia prolongada en la UMI y la insuficiencia renal. Por otra parte, los pacientes en peor situación previa - mala CVRS previa o enfermedades crónicas recogidas por el APACHE II- que han sobrevivido a los 6 meses muestran un menor deterioro de su CVRS.

\section{BIBLIOGRAFÍA}

1. Jakob SM, Rothen HU. Intensive care 1980-1995. Change in patient characteristics, nursing worload and outcome. Intensive Care Med. 1997;23:1165-70.

2. Teres D, Rapoport J. Identifying patients with high risk of high cost. Chest. 1991;99:530-1.

3. Consensus conference organized by the ESICM and the SRLF. Predicting outcome in ICU patients. Intensive Care Med. 1994;20:390-7.

4. Spanish Group for Epidemiological Analysis of Critical Patients. Quality of life: a tool for decision-making in the ICU. Intensive Care Med. 1994;20:251-2.

5. Hennessy D, Juzwishin K, Yergens D, Noseworthy T, Doig C. Outcomes of elderly survivors of intensive care. A review of the literature. Chest. 2005;127:1764-74.

6. The Society of Critical Care Medicine Ethics Committee. Attitudes of critical care medicine professionals concerning distribution of intensive care resources. Crit Care Med. 1994;22:358-62.

7. Conferencia de Consenso sobre EPOC. Arch Bronconeumol. 2003;Supl 3:1-47.

8. Knaus WA, Draper EA, Wagner DP, Zimmermen JE. APACHE II: a severity of disease clasification system. Crit Care Med. 1985;13:818-29.

9. Bone RC, Balk RA, Cerra FB, Dellinger RP, Fein AM, Knaus WA, et al. Definitions for sepsis and organ failure and guidelines for the use of innovative therapies in sepsis. The ACCP/ SCCM Consensus Conference Committee. American College of Chest Physicians/Society of Critical Care Medicine. Chest. 1992;101:1644-55.

10. Vincent JL, Moreno R, Takala J, Willatts S, De Mendoça A, Bruining H, et al. The SOFA (Sepsis-related Organ Failure Assessment) score to describe organ dysfunction/failure. On behalf of the Working Group on Sepsis-Related Problems of the European Society of Intensive Care Medicine. Intensive Care Med. 1996;22:707-10

11. Moreno R, Vincent JL, Matos R, Mendoça A, Cantraine $\mathrm{F}$, Thijs L, et al; on behalf of the working group on sepsisrelated problems of the ESICM. The use of maximum SOFA score to quantify organ dysfunction/failure in intensive care. Results of a prospective, multicentre study. Intensive Care Med. 1999;25:68695.

12. Fernandez RR, Cruz JJ, Mata GV, PAEEC. Validation of a quality of life questionnaire for critically ill patients. Intensive Care Med. 1996;22:1034-42.

13. Iribarren-Diarasarri S, Aizpuru-Barandiaran F, Loma-Osorio A, Castedo-González J, Poveda-Hernández Y, Muñoz-Martínez T, et al. Factores pronósticos de mortalidad en pacientes con enfermedad pulmonar obstructiva crónica tras su ingreso en una Unidad de Medicina Intensiva. El papel de la Calidad de Vida. Med Intensiva. 2005;29:204-11.

14. Iribarren-Diarasarri S, Latorre-García K, Muñoz-Martínez T, Poveda-Hernández Y, Dudagoitia-Otaolea JL, Martínez Alutiz $\mathrm{S}$, et al. Limitación del esfuerzo terapéutico tras el ingreso en una Unidad de Medicina Intensiva. Análisis de factores asociados. Med Intensiva. 2007;31:68-72.

15. Capuzzo M, Graselli Cl, Carrer S, Gritti G, Alvisi R. Validation of two quality of life questionnaires suitable for intensive care patients. Intensive Care Med. 2000;26:1296-303. 
16. Gopal I, Bhonagiri S, Ronco C, Bellomo R. Out of hospital outcome and quality of life in survivors of combined acute multiple organ and renal failure treated with continuous venovenous hemofiltration/hemodiafiltration. Intensive Care Med. 1997;23:766-72.

17. Hurel D, Loirat P, Saulnier F, Nicolas F, Brivet F. Quality of life 6 months after intensive care: results of a prospective multicenter study using a generic health status scale and a satisfaction scale. Intensive Care Med. 1997; 23:331-7.

18. Badia X, Diaz-Prieto A, Rue M, Patrick DL. Measuring health and health state preferences among critically ill patients. Intensive Care Med. 1996;22:1379-84.

19. Ambrosino N, Bruletti G, Scala V, Porta R, Vitacca M. Cognitive and perceived health status in patient with chronic obstructive pulmonary disease surviving acute on chronic respiratory failure: a controled study. Intensive Care Med. 2002;28:170-7.

20. Yinnon A, Zimran A, Hershko C. Quality of life and survival following intensive medical care. Q J Med. 1989;71:347-57.

21. Konopad E, Noseworthy Th, Johnston R, Shustack A, Grace M. Quality of life measures before and one year after admission to an intensive care unit. Crit Care Med. 1995;23:1653-9.

22. Niskanen M, Ruokonen E, Takala J, Rissanen P, Kari A. Quality of life after prolonged intensive care. Crit Care Med. 1999;27:1132-9.

23. Pettilä V, Kaarlola A, Mäkeläinen A. Health-related quality of life of multiple organ dysfunction patients one year after intensive care. Intensive Care Med. 2000;26:1473-9.

24. Combes A, Costa MA, Trouillet JL, Baudot J, Mokhtari M, Gibert CL, et al. Morbidity, mortality, and quality-of-life outcomes of patients requiring $\geq 14$ days of mechanical ventilation. Crit Care Med. 2003;31:1373-81.

25. Rivera-Fernández R, Navarrete-Navarro $P$, FernándezMondejar E, Rodríguez-Elvira M, Guerrero-López F, VázquezMata G; Project for the Epidemiological Analysis of Critical Care Patients (PAEEC) group. Six-year mortality and quality of life in critically ill patients with chronic obstructives pulmonary disease. Crit Care Med. 2006;34:2317-24.

26. Badía Castelló M, Trujillano Cabello J, Serviá Goixart L, March Llanes J, Rodríguez Pozo A. Cambios en la calidad de vida tras UCI según grupo diagnóstico. Comparación de dos instrumentos de medida. Med Intensiva. 2008:32:203-15.

27. Vázquez Mata G, Rivera Fernández R, González Carona A, Delgado-Rodríguez M, Torres Ruiz JM, Raya Pugnaire A, et al.
Factors related to quality of life 12 months after discharge from an intensive care unit. Critical Care Med. 1992;20:1257-62.

28. Wehler M, Geise A, Hadzionerovic D, Aljukic E, Reulbach U, Hahn EG, et al. Health-related quality of life of patients with multiple organ dysfunction: individual changes and comparison with normative population. Crit Care Med. 2003;31:1094-101.

29. Cuthbertson BH, Scott J, Strachan M, Kilonzo M, Vale L. Quality of life before and after intensive care. Anaesthesia. 2005; 60:332-9.

30. Rivera-Fernández R, Sánchez-Cruz JJ, Abizanda-Campos R, Vázquez-Mata G. Quality of life before intensive care unit admission and its influence on resource utilization and mortality rate. Crit Care Med. 2001:29:1701-9.

31. Clermont G, Angus DC, Linde-Zwirble WT, Griffin MF, Fine MJ, Pinsky MR. Does acute organ dysfunction predict patient-centered outcomes? Chest. 2002;121:1963-71.

32. Graf J, Koch M, Dujardin R, Kersten A, Janssens U. Healthrelated quality of life before, 1 month after, and 9 months after intensive care in medical cardiovascular and pulmonary patients. Crit Care Med. 2003;31:2163-9.

33. Korkeila M, Ruokonen E, Takala J. Costs of care, long-term prognosis and quality of life in patients requiring renal replacement therapy during intensive care. Intensive Care Med. 2000;26: 1824-31.

34. Capuzzo M, Moreno RP, Jordan B, Bauer P, Alvisi R, Metnitz PG. Predictors of early recovery of health status alter intensive care. Intensive Care Med. 2006;32:1832-8.

35. Garnacho-Montero J, Madrazo-Osuna J, García-Garmendia JL, Ortiz-Leyba C, Jiménez-Jiménez FJ, Barrero-Almodóvar A, et al. Critical illness polyneuropathy: risk factors and clinical consequences. A cohort study in septic patients. Intensive Care Med. 2001:27:1288-96.

36. Van der Schaaf M, Beelen A, De Vos R. Functional outcome in patients with critical illness polyneuropathy. Disabil Rehabil. 2004;26:1189-97.

37. Welsh CH, Thompson K, Long-Krug S. Evaluation of patient-perceived health status using the Medical Outcomes Survey Short-Form 36 in an intensive care unit population. Crit Care Med. 1999:27:1466-71.

38. Capuzzo M, Grasselli C, Carrer S, Gritti G, Alvisi R. Quality of life before intensive care admission: agreement between patient and relative assessment. Intensive Care Med. 2000;26:1288-95. 